\title{
Rehabilitación de un suelo salino con yeso agrícola en un cultivo de nogal en el Valle del Yaqui
}

\section{Rehabilitation of a saline soil with gypsum in a pecan orchard in the Yaqui Valley}

\author{
Alma Trasviña Barriga ${ }^{1}$, Rafael Bórquez Olguín ${ }^{1}$, José Leal Almanza ${ }^{1}$, \\ Luciano Castro Espinoza ${ }^{1}$ y Marco Gutiérrez Coronado ${ }^{1 \ddagger}$
}

\footnotetext{
${ }^{1}$ Instituto Tecnológico de Sonora. 5 de Febrero 818 Sur, Col. Centro. 85000 Ciudad Obregón, Sonora, México.

`Autor responsable (mgutierrez@itson.edu.mx)
}

\section{RESUMEN}

El cultivo de nogal es una actividad en amplio crecimiento en el Valle del Yaqui, Sonora. Las zonas nogaleras de la región se caracterizan por presentar problemas de salinidad y altas concentraciones de sodio intercambiable, lo que provoca disminución en la productividad de la nuez pecana. Al terminar la temporada de riego con agua del sistema de presas del Río Yaqui, el uso de agua de pozos profundos es utilizada, generalmente en los meses de mayo a agosto. Esto puede ocasionar acumulación de sales en el suelo cuando el agua es de mala calidad. El yeso agrícola ha sido utilizado como enmienda para rehabilitar el suelo mejorando las propiedades químicas del mismo. El objetivo del presente estudio fue determinar el efecto de la aplicación de yeso agrícola en un suelo salino de un huerto de nogal en el Valle del Yaqui. Se llevó a cabo la rehabilitación durante dos años, en el primer año se emplearon dosis de 5 y $10 \mathrm{Mg} \mathrm{ha}^{-1}$, mientras que en el segundo año $2 \mathrm{Mg} \mathrm{ha}^{-1}$ de yeso agrícola. Se realizaron dos lavados por melgas después de cada aplicación. Los análisis químicos del suelo se ejecutaron de acuerdo a la NOM-021-RECNAT-2000 durante tres años, previo y posterior a las aplicaciones de enmienda. Posterior a la aplicación de enmienda, disminuyeron los valores de conductividad eléctrica de 12.41 a $6.29 \mathrm{dS} \mathrm{m}^{-1}$, porcentaje de sodio intercambiable de 12.48 a 5.57 y relación de adsorción de sodio de 10.54 a 4.88 en la profundidad de 0-30 $\mathrm{cm}$ en la primera aplicación. El empleo de yeso agrícola y los lavados en el suelo salino del huerto de nogal mejoraron las propiedades químicas.

Cita recomendada:

Trasviña Barriga, A., R. Bórquez Olguín, J. Leal Almanza, L. Castro Espinoza y M. Gutiérrez Coronado. 2018. Rehabilitación de un suelo salino con yeso agrícola en un cultivo de nogal en el Valle del Yaqui. Terra Latinoamericana 36: 85-90.

DOI: https://doi.org/10.28940/terra.v36i1.310
Palabras clave: sulfato de calcio, PSI, conductividad eléctrica, lavado de suelo, $\mathrm{pH}$.

\section{SUMMARY}

Pecans are cultivated extensively in the Yaqui Valley, Sonora. These soils often have problems of salinity and high concentrations of exchangeable sodium, which cause a decline in pecan productivity. At the end of the season of irrigation with water from the system of reservoirs on the Yaqui River, groundwater is generally used from May to August. This can result in salt accumulation in the soil when water is of poor quality. Gypsum has been used as an amendment to rehabilitate soil by improving its physical and chemical properties. The main objective of this study was to investigate the effect of gypsum application to a saline soil of a pecan orchard in the Yaqui Valley. Rehabilitation was carried out for two consecutive years. In the first year a dose of 5 and $10 \mathrm{Mg} \mathrm{ha}^{-1}$ was applied, while in the second year $2 \mathrm{Mg} \mathrm{ha}^{-1}$ of gypsum was used. Two ridge washings were done after each application. The physico-chemical analyses of the soil were conducted following NOM-021-RECNAT-2000 during three years prior to and after the applications of amendment. Once the amendment was applied, the values of electrical conductivity decreased from 12.41 to $6.29 \mathrm{dS} \mathrm{m}^{-1}$, percentage of exchangeable sodium from 12.48 to 5.57 and sodium adsorption ratio from 10.54 to 4.88 at the depth of $0-30 \mathrm{~cm}$. The use of agricultural gypsum and washing in a saline soil of the pecan orchard improved soil chemical properties using 5 and $10 \mathrm{Mg} \mathrm{ha}^{-1}$.

Recibido: abril de 2017. Aceptado: diciembre de 2017. Publicado como nota de investigación en Terra Latinoamericana 36: 85-90. 
Index words: calcium sulfate, ESP, electrical conductivity, soil washing, $\mathrm{pH}$.

\section{INTRODUCCIÓN}

El cultivo de nuez pecanera en México es una actividad en crecimiento, los estados de mayor producción se encuentran al norte del país (Zaragoza-Lira et al., 2011). Los suelos de México se ven afectados por concentraciones de salinidad en un 3.2\% del territorio (Mata Fernández et al., 2014). Las actividades agrícolas se ven desfavorecidas con los problemas de salinidad y altos niveles de sodio intercambiable (Rivera et al., 2014). Las sales en la planta afectan los procesos bioquímicos y la retención de agua (Mata Fernández et al., 2014; Smith et al., 2015). A lo largo de los años, se ha aplicado el sulfato de calcio di hidratado $\left(\mathrm{CaSO}_{4} 2 \mathrm{H}_{2} \mathrm{O}\right)$ comúnmente llamado yeso agrícola, el cual es de bajo costo, como enmienda para el suelo. Dicho manejo ha demostrado el mejoramiento de las propiedades físicas y químicas del suelo, así como el aumento de la productividad de los cultivos (Rasouli et al., 2013; Yazdanpanah y Mahmoodabadi, 2013). El mecanismo de acción del yeso agrícola, es el reemplazo del calcio por el sodio del suelo mediante intercambio catiónico, para formar sulfato de sodio el cual es lixiviado (Sibbett, 1995). Bajo esta perspectiva, el objetivo del presente estudio fue evaluar el efecto en las propiedades químicas de la aplicación de yeso agrícola en un suelo salino en el Valle del Yaqui.

\section{MATERIALES Y MÉTODOS}

El sitio experimental fue un huerto comercial de nogal pecanero de 50 ha. Localizado en el Valle del Yaqui, bloque 1502 con coordenadas $27^{\circ} 14$ ' 48.6" $\mathrm{N}$ y $110^{\circ} 00^{\prime} 34.9^{\prime \prime} \mathrm{O}$. Fue plantado en el 2010 con un marco real de $8 \times 8$ de las variedades Western Schley y Wichita. Cuenta con sistema de riego por goteo y drenaje parcelario a $1.40 \mathrm{~m}$. Los análisis de suelo se efectuaron en las instalaciones del Instituto Tecnológico de Sonora en el laboratorio de Análisis de agua, suelo y planta.

\section{Aplicación de Yeso Agrícola}

La aplicación de enmienda se llevó a cabo en diciembre de 2015 y 2016. La parcela se dividió en dos zonas según el estado de los árboles (necrosis $\mathrm{y}$ amarillamiento) y las sales visibles en suelo.
En el año 2015 a la zona 1 se le aplicó una dosis de $5 \mathrm{Mg} \mathrm{ha}^{-1}$ de yeso agrícola, mientras que a la zona 2, $10 \mathrm{Mg} \mathrm{ha}^{-1}$. En el año 2016 se aplicó la dosis de $2 \mathrm{Mg} \mathrm{ha}^{-1}$ a las dos zonas. El yeso agrícola fue incorporado con un paso de rastra después de su aplicación terrestre con cajón, previo a ello se llevó a cabo la preparación del suelo con cincel y rastra seguido del levantamiento del sistema de riego por inundación controlada (melgas). Se llevó a cabo un riego de humedecimiento y se dejó actuar el yeso agrícola sobre el suelo. Después se realizaron dos riegos por inundación por melgas. Con duración de 12 horas en el mes de diciembre y enero.

\section{Muestreo y Análisis Químicos del Suelo}

Se realizaron tres análisis de suelo del huerto de nogal durante tres años consecutivos. Uno previo a la aplicación de enmienda en el mes de junio de 2015 y posterior de cada aplicación en el mes de abril de 2016 y 2017. Los pozos se realizaron al centro de la calle. El muestreo inicial se realizó en cuatro puntos de la parcela a tres profundidades $(0-30,30-60$ y 60-100 cm). El muestreo de 2016 se efectuó en seis puntos. Mientras que, en 2017, se obtuvieron dos muestras compuestas de tres pozos cada uno para cada zona, a las profundidades de $0-30$ y $30-60 \mathrm{~cm}$. Los análisis de suelo se llevaron a cabo de acuerdo con la NOM-021-RECNAT-2000. El primero fue previo a la aplicación de enmienda en el mes de mayo de 2015. Se contemplaron los análisis de \% de materia orgánica, textura, $\mathrm{pH}$, conductividad eléctrica (CE), relación de absorción de sodio (RAS) y porciento de sodio intercambiable (PSI). Para el segundo y tercer análisis, en el mes de mayo de 2016 y 2017, se realizaron los análisis de $\mathrm{pH}, \mathrm{CE}$, RAS y PSI.

\section{Análisis Estadístico}

Se realizó un análisis de varianza. Para la diferencia entre las medias de cada profundidad se utilizó la prueba de $\operatorname{LSD}(P \leq 0.05)$, con el programa estadístico STATGRAPHICS Plus 5.1.

\section{RESULTADOS Y DISCUSIÓN}

\section{Características del Agua de Pozo Utilizada para Riego}

En los meses de mayo a agosto el agua que es utilizada proviene de un pozo localizado en 
el predio. Los análisis de calidad del agua mostraron una CE fue de $1978.00 \mu \mathrm{S} \mathrm{cm}^{-1}$, ha sido clasificada como salinidad de media a elevada y el pH es de 6.61 (Cuadro 1).

\section{Características del Suelo del Huerto de Nogal}

Los resultados de los análisis químicos, previo a la aplicación de enmienda se muestran en el Cuadro 2. Se clasificó al suelo del huerto de nogal como salino, según los criterios de la NOM-021-RECNAT-2000.

\section{Textura}

El suelo del huerto de nogal se clasificó como un suelo arcilloso (Cuadro 3). Éstos se caracterizan por su baja permeabilidad y baja conductividad hidráulica (Ravinder et al., 2017).

\section{Materia Orgánica}

La media para la materia orgánica en el suelo donde está establecida la huerta de nogal fue de $1.71 \%$ para la profundidad de 0 a $30 \mathrm{~cm}$; de acuerdo a la NOM-021-RECNAT-2000 estos suelos se clasifican de nivel medio de materia orgánica.

\section{pH}

Los valores de $\mathrm{pH}$ para el huerto de nogal fueron de 7.52 a 7.92 (Figura 1). De acuerdo a la NOM-021-RECNAT-2000, el suelo se clasifica como medianamente alcalino. La mayoría de los cultivos de nuez se obtienen en suelos con $\mathrm{pH}$ de 5.5 a 7 (Sibbett, 1995). El pH está relacionado con la absorción y la disponibilidad de los nutrientes para

Cuadro 1. Concentración de cationes y aniones en el agua del pozo.

\begin{tabular}{lc}
\hline Catión/Anión & Concentración \\
\hline & $\mathrm{mg} \mathrm{L}^{-1}$ \\
Calcio & 116.23 \\
Magnesio & 53.50 \\
Sodio & 220.07 \\
Cloruros & 444.43 \\
Sulfatos & 227.23 \\
Carbonatos & 0.00 \\
Bicarbonatos & 153.07 \\
\hline
\end{tabular}

Cuadro 2. Propiedades químicas del suelo del huerto de nogal.

\begin{tabular}{lcccc}
\hline Profundidad & $\mathrm{pH}$ & $\mathrm{CE}$ & $\mathrm{RAS}$ & PSI \\
\hline $\mathrm{cm}$ & \multicolumn{5}{c}{$\mathrm{dS} \mathrm{m}^{-1}$} \\
$0-30$ & 7.52 & 12.42 & 10.54 & 12.48 \\
$30-60$ & 7.68 & 5.24 & 7.48 & 8.85 \\
$60-100$ & 7.83 & 4.13 & 4.79 & 5.46 \\
\multicolumn{5}{l}{} \\
CE = conductividad eléctrica; RAS = relación de absorción de sodio; \\
PSI porciento de sodio intercambiable.
\end{tabular}

la planta, sobre todo de fósforo y micronutrientes (Ramos-Leal et al., 2016). Posterior a la aplicación de yeso agrícola el pH aumentó sólo de 0-30 y $30-60 \mathrm{~cm}$. Cierta cantidad de sodio pudo haberse hidrolizado y causar el incremento.

\section{Conductividad Eléctrica}

Los valores de conductividad eléctrica disminuyeron de $12.42 \mathrm{dS} \mathrm{m}^{-1}$ a 6.29 y $7.3 \mathrm{dS} \mathrm{m}^{-1}$ al realizar la aplicación de 5 y $10 \mathrm{Mg} \mathrm{ha}^{-1}$ de yeso a la profundidad de 0-30 cm (Figura 2). Este compuesto promueve una reacción de intercambio catiónico. $\mathrm{El} \mathrm{Ca}^{2+}$ por el $\mathrm{Na}^{+}$del suelo, lleva a la formación de sulfato de calcio. El cual puede ser eliminado mediante lavados debido a su alta solubilidad. La NOM-021-RECNAT-2000 clasifica a los suelos como salinos cuando poseen una conductividad

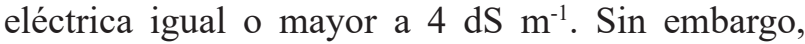
estudios previos indican que valores mayores a $3 \mathrm{dS} \mathrm{m}^{-1}$ tienden a reducir el área transversal del tronco y el rendimiento por árbol (Santamaría et al., 2002). En la mayoría de los muestreos, a excepción de los de 30-100 $\mathrm{cm}$ de profundidad se obtuvieron valores mayores a $4 \mathrm{dS} \mathrm{m}^{-1}$. Estos valores son similares a los reportados por Zaragoza-Lira et al. (2011) en el estado de Coahuila, quien encontró para las profundidades de 0-30, 30-60 y 60-90 cm valores de 4.3, 5 y $5.3 \mathrm{dS} \mathrm{m}^{-1}$, respectivamente. Y menores a los reportados por Arellano-Gil y Gutiérrez-

Cuadro 3. Fracciones granulométricas del huerto de nogal.

\begin{tabular}{|c|c|c|c|}
\hline Profundidad & Arena & Arcilla & Limo \\
\hline $\mathrm{cm}$ & $\ldots \ldots$ & $-\%-$ & $-\ldots$ \\
\hline $0-30$ & 13.04 & 66.11 & 20.85 \\
\hline $30-60$ & 12.68 & 69.11 & 18.21 \\
\hline $60-100$ & 9.72 & 71.61 & 18.67 \\
\hline
\end{tabular}




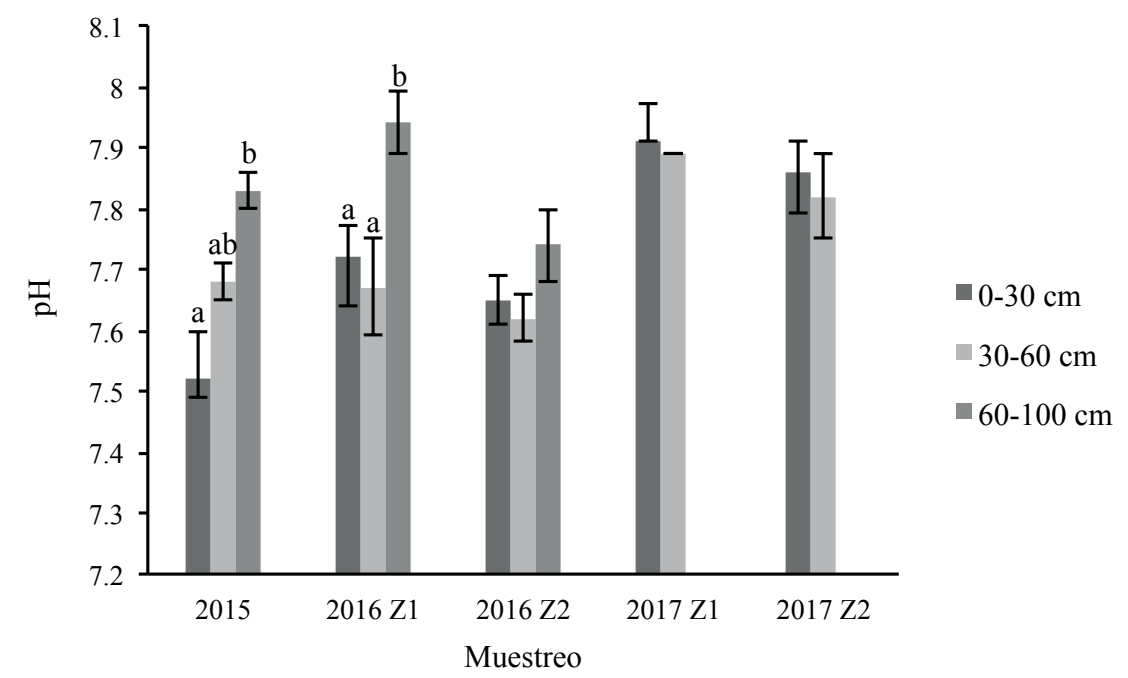

Figura 1. Efecto de la aplicación de yeso agrícola sobre el pH en el huerto de nogal. Valores de medias de $\mathrm{pH} \pm$ error estándar. Z1 corresponde a la zona de aplicación de $5 \mathrm{Mg} \mathrm{ha}^{-1}$, mientras que la Z2 a $10 \mathrm{Mg} \mathrm{ha}^{-1}$ de yeso agrícola en el año 2016.

Coronado (2006) que fueron de 2.1 y $2 \mathrm{dS} \mathrm{m}^{-1}$ para las profundidades de 0-15 y 15-30 respectivamente en el Valle del Yaqui, Sonora. La conductividad eléctrica puede permanecer en rangos temporalmente estables durante dos temporadas (Stadler et al., 2015). Sin embargo, puede haber acumulación de sales de un año a otro debido a las condiciones del agua de riego (Grageda et al., 2011). Lo que pudiera ser razón del aumento de CE del año 2017 y la disminución de dosis de yeso agrícola a $2 \mathrm{Mg} \mathrm{ha}^{-1}$.

\section{Relación de Adsorción de Sodio}

Los valores de RAS disminuyeron posterior a la aplicación de yeso agrícola a excepción de la zona 2 en el año 2017. (Figura 3). En 2015 se encontraron los valores más altos, de 12.48 disminuyó a 5.57 y 6.2 en el año 2016 para la zona 1 y 2 en la profundidad de $0-30 \mathrm{~cm}$. Esto se puede deber al intercambio de $\mathrm{Na}$ por $\mathrm{Ca}$ en las partículas de arcilla en suelo debido al efecto de la aplicación de yeso agrícola. En la zona 2

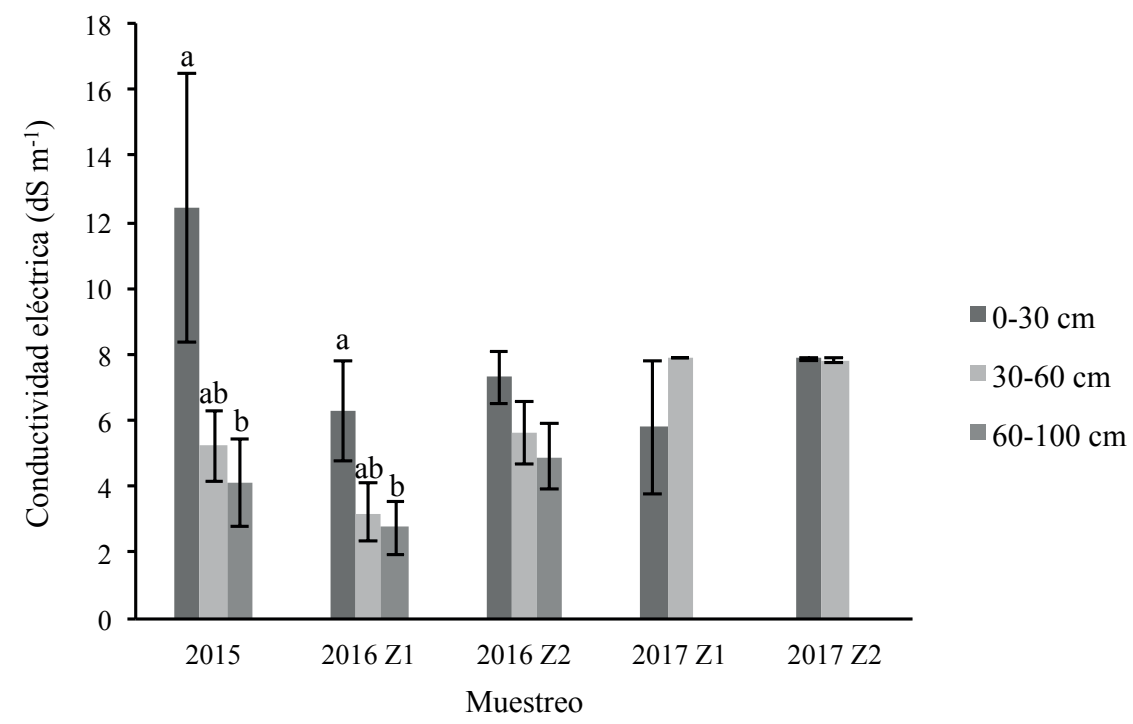

Figura 2. Efecto de la aplicación de yeso agrícola en la conductividad eléctrica. Valores de medias de $\mathrm{CE} \pm$ error estándar. Z1 corresponde a la zona de aplicación de $5 \mathrm{Mg} \mathrm{ha}^{-1}$, mientras que la Z2 a $10 \mathrm{Mg} \mathrm{ha}^{-1}$ de yeso agrícola en el año 2016. 


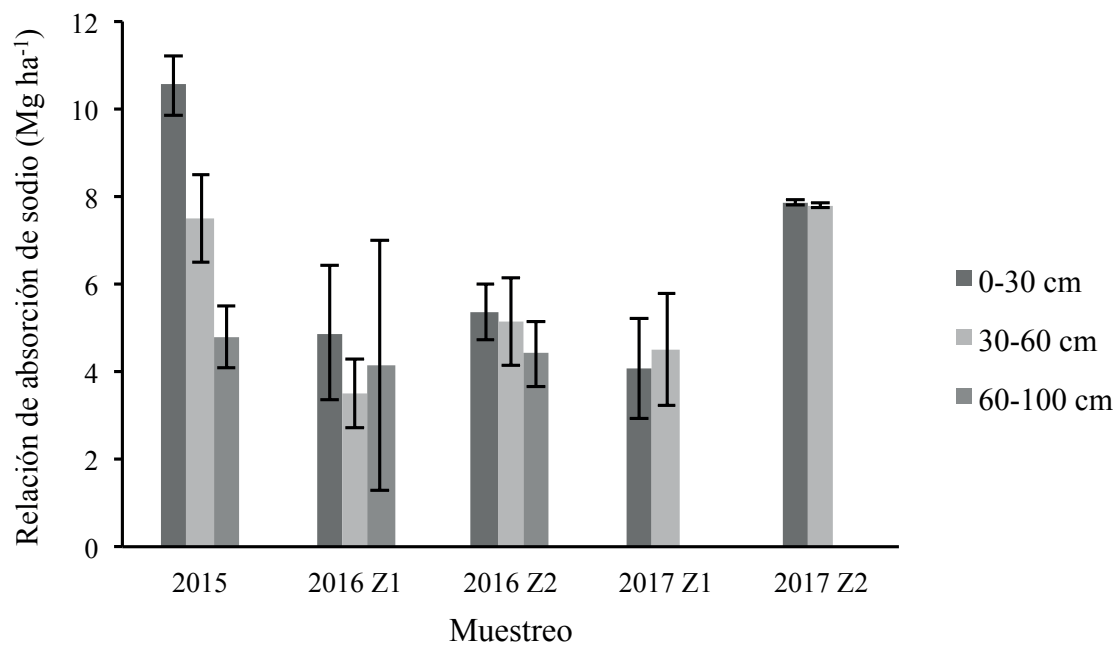

Figura 3. Efecto de la aplicación de yeso agrícola en la relación adsorción sodio. Valores de medias de RAS \pm error estándar. Z1 corresponde a la zona de aplicación de $5 \mathrm{Mg} \mathrm{ha}^{-1}$, mientras que la Z2 a $10 \mathrm{Mg} \mathrm{ha}^{-1}$ de yeso agrícola en el año 2016.

correspondiente a la zona de aplicación de $10 \mathrm{Mg} \mathrm{ha}^{-1}$ de yeso agrícola, se encontraron valores mayores en comparación con la zona 1 de $5 \mathrm{Mg} \mathrm{ha}^{-1}$, lo que indica una acumulación de sales mayor, el mismo efecto se obtuvo en el año 2017.

\section{Porcentaje de Sodio Intercambiable}

En el año 2015 se obtuvieron los valores más altos de PSI en las tres profundidades. Éstos disminuyeron tras la aplicación de yeso agrícola (Figura 4). Estos resultados siguen la tendencia que los obtenidos en RAS, debido al efecto del lavado del sodio por efecto del yeso agrícola en el suelo. Según la NOM-021RECNAT-2000 un suelo sódico es aquel que tiene valores de 15 PSI o más. Sin embargo, Santamaría et al. (2002) reportaron que a valores superiores a $3 \%$ existe un efecto negativo en el tronco y en rendimiento de nuez por árbol; en cambio, tiende a reducir la alternancia del nogal.

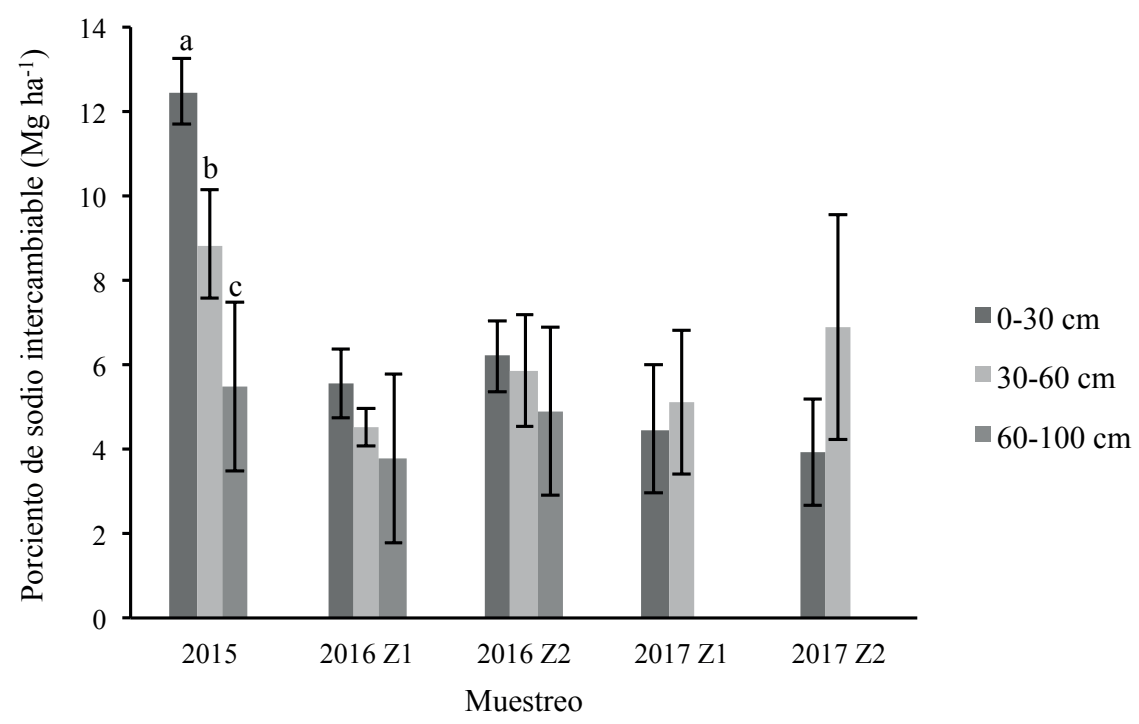

Figura 4. Efecto de la aplicación de yeso agrícola en el PSI. Valores de medias de PSI \pm error estándar. Z1 corresponde a la zona de aplicación de $5 \mathrm{Mg} \mathrm{ha}^{-1}$, mientras que la Z2 a $10 \mathrm{Mg} \mathrm{ha}^{-1}$ de yeso agrícola en el año 2016. 


\section{CONCLUSIONES}

La aplicación de yeso agrícola y los lavados en el suelo salino mejoraron las propiedades químicas del mismo. Asimismo, disminuyeron los valores de CE, RAS y PSI posterior a las aplicaciones de enmienda en el año 2016 con las dosis de 5 y $10 \mathrm{Mg} \mathrm{ha}^{-1}$ de yeso agrícola. El pH aumentó posterior a la aplicación de tratamientos.

\section{LITERATURA CITADA}

Arellano-Gil, M.y M.A. Gutiérrez-Coronado. 2006. Rendimiento y calidad poscosecha de tomate bajo diferentes esquemas de fertilización al suelo. Rev. Chapingo Serie Hortic. 12: 113-118.

Grageda, J., R. Sabori, A. Valenzuela, A. Quijada, J. H. Núñez y J. C. Rodríguez. 2011. Salinidad del suelo en huertas de nogal pecanero Carya illinoinensis (Wangenh.) K. Koch. Rev. Cienc. Biol. Salud 8: 22-27.

Mata Fernández, I., M. L. Rodríguez G., J. López B. y G. Vela C. 2014. Dinámica de la salinidad en los suelos. Rev. Digital Depto. Hombre Amb. 1: 26-35.

NOM-021-RECNAT-2000 (NORMA Oficial Mexicana). 2000. Que establece las especificaciones de fertilidad, salinidad y clasificación de suelos. Estudios, muestreo y análisis. Secretaría de Medio Ambiente y Recursos Naturales. México, D. F.

Ramos-Leal, J. A., B. López-Álvarez, G. Santacruz-De León, O. Almanza-Tovar, J. Morán- Ramírez, D. A. PadillaReyes, and Z. I. González-Acevedo. 2016. Quality indices of groundwater for agricultural use in the region of Tierra Nueva, San Luis Potosi, Mexico. Arab. J. Geosci. 9: 736. doi:10.1007/s12517-016-2758-2.

Rasouli, F., A. K. Pouya, and N. Karimian. 2013. Wheat yield and physico-chemical properties of a sodic soil from semiarid area of Iran as affected by applied gypsum. Geoderma 193-194: 246-255. doi:10.1016/j.geoderma.2012.10.001.
Ravinder, J., T. Lokya, N. M. Konde, and P. Thakur. 2017. Potentiality of BFF and soil amendments in degraded purnavalley soils reclamation and soybean yield. Int. J. Curr. Microbiol. Appl. Sci. 6: 2540-2548. doi: 10.20546/ ijcmas.2017.606.302.

Rivera G., M., M. Palomo R., G. Delgado R., J. Estrada A. y J. A. Muñoz V. 2014. Evaluación de la tolerancia a salinidad y sodicidad de cuatro especies de pasto, en un suelo salino sódico de un bosque urbano. Agrofaz 14: 79-82.

Santamaría, C., J. Medina M., M. C. Rivera G. y R. Faz C. 2002. Algunos factores de suelo, agua y planta que afectan la producción y alternancia del nogal pecanero. Rev. Fitotec. Mex. 25: 119-125.

Sibbett, G. 1995. Managing high pH, calcareous, saline, and sodic soils of the Western Pecan-growing region. HortTechnology 5: 222-225.

Smith, P., M. F. Cotrufo, C. Rumpel, K. Paustian, P. J. Kuikman, J. A. Elliott, R. McDowell, R. I. Griffiths, S. Asakawa, M. Bustamante, J. I. House, J. Sobocká, R. Harper, G. Pan, P. C. West, J. S. Gerber, J. M. Clark, T. Adhya, R. J. Scholes, and M. C. Scholes. 2015. Biogeochemical cycles and biodiversity as key drivers of ecosystem services provided by soils. Soil 1: 665-685. doi:10.5194/soil-1-665-2015.

Stadler, A., S. Rudolph, M. Kupisch, M. Langensiepen, J. van der Kruk, and F. Ewert. 2015. Quantifying the effects of soil variability on crop growth using apparent soil electrical conductivity measurements. Eur. J. Agron. 64: 8-20. doi: 10.1016/j.eja.2014.12.004.

STATGRAPHICS. 2016. STATGRAPHICS Plus Professional (v. 5.1) - box pack. Manugistics Group. New York, NY, USA.

Yazdanpanah, N. and M. Mahmoodabadi. 2013. Reclamation of calcareous saline-sodic soil using different amendments: Time changes of soluble cations in leachate. Arab. J. Geosci. 6: 2519-2528. doi:10.1007/s12517-011-0505-2.

Zaragoza-Lira, M. M., P. Preciado-Rangel, U. FigueroaViramontes, J. L. García-Hernández, M. Fortis-Hernández, M. A. Segura-Castruita, A. Lagarda-Murrieta y E. MaderoTamargo. 2011. Aplicación de composta en la producción del nogal pecanero. Rev. Chapingo Serie Hortic. 17: 33-37. doi:10.1016/j.chemosphere.2005.12.057. 